\title{
Renal expression of advanced oxidative protein products predicts progression of renal fibrosis in patients with IgA nephropathy
}

\author{
Jun Wang ${ }^{1}$, Min Liang ${ }^{1}$, Jie Xu, Wei Cao, Guo B Wang, Zhan M Zhou, Jian W Tian, Nan Jia, Zhenhai Zhang, Jing Nie, \\ Youhua Liu and Fan F Hou
}

Predicting the risk of disease progression in IgA nephropathy (IgAN) remains a challenge. This study was conducted to test the hypothesis that renal accumulation of advanced oxidized protein products (AOPPs) is an early predictor for renal progression in IgAN. This was a single-center prospective cohort study. One hundred IgAN patients with eGFR $>80 \mathrm{ml} / \mathrm{min} / 1.73 \mathrm{~m}^{2}$ were enrolled. Seventy-seven patients were followed for a mean of 4.2 years, and 30 patients received repeat renal biopsy at a mean of 42 months after diagnosis. The outcomes were the progression of renal fibrosis and rapid progression of CKD ( $>5 \mathrm{ml} / \mathrm{min} / 1.73 \mathrm{~m}^{2} /$ year) during follow-up. Immunoreactivity of AOPPs was detected predominantly in tubular epithelial cells and co-localized with expression of TGF- $\beta 1$ and angiotensin II. Renal staining score of AOPPs at diagnosis was associated with the level of tissue cellular inflammation. Accumulation of AOPPs, particularly in interstitial-infiltrating cells, was negatively correlated with changes of eGFR during follow-up; those with expression scores greater than the median at diagnosis had significantly higher incidences of rapid decline of eGFR compared with those with the score less than or equal to the median. For patients who received repeat renal biopsy, renal AOPP levels greater than the median at diagnosis were associated with increase in renal fibrosis index at repeat biopsy. After multivariate adjustment, renal AOPP expression was an independent predictor for progression of renal fibrosis and rapid decline of eGFR. Taken together, these results demonstrate that renal AOPPs might be a predictor, detectable at the time of diagnosis, for renal progression in patients with early stage IgAN.

Laboratory Investigation (2014) 94, 966-977; doi:10.1038/labinvest.2014.90; published online 28 July 2014

Immunoglobulin A nephropathy (IgAN) is the most prevalent type of primary glomerulonephritis and the leading cause of end-stage renal disease (ESRD), especially in the Asia-Pacific area. It is recognized that the outcome of IgAN is highly variable among individuals. The progression of IgAN to ESRD is reported to vary from 5 to $25 \%$ after 10 years and $25-50 \%$ after 20 years, with a spontaneous clinical remission rate from 5 to $30 \% .{ }^{1}$ Therefore, early identification of patients at high risk of renal progression, particularly at the time of diagnosis, would facilitate timely interventions and avoid unnecessary exposure of toxic therapies.

Several clinical variables have consistent and independent associations with worse kidney prognosis. Increased serum creatinine, arterial hypertension, and nephrotic-range proteinuria at discovery are meaningful but not powerful predictors of an adverse long-term outcome. The levels of blood pressure and urinary protein excretion during followup are more predictive on outcome. ${ }^{2}$ An additional predictor of an adverse renal outcome is an elevated IgA/C 3 concentration ratio at baseline, but this has not yet been confirmed in multiple, geographically diverse populations. ${ }^{3}$

Renal fibrosis is a common pathway for progression of chronic kidney disease (CKD). Glomerulosclerosis and interstitial fibrosis has been identified as strong predictors of outcome. However, these histological lesions may not be obvious in early IgAN. ${ }^{4}$ Our ability to reliably riskstratify patients with IgAN at the time of diagnosis remains limited.

National Clinical Research Center of Kidney Disease, State Key Laboratory of Organ Failure Research, Guangdong Provincial Institute of Nephrology, Division of Nephrology, Nanfang Hospital, Southern Medical University, Guangzhou, P.R. China

Correspondence: Professor FF Hou, National Clinical Research Center of Kidney Disease, State Key Laboratory of Organ Failure Research, Guangdong Provincial Institute of Nephrology, Division of Nephrology, Nanfang Hospital, Southern Medical University, 1838 North Guangzhou Avenue, Guangzhou 510515, P.R. China.

E-mail: ffhouguangzhou@163.com

${ }^{1}$ These two authors contributed equally to this work.

Received 12 December 2013; revised 24 April 2014; accepted 29 May 2014 
Activation of the oxidative stress pathway has been identified in IgAN. Imbalance of oxidation/antioxidation has been detected in sera and/or in erythrocytes of patients with IgAN,${ }^{5}$ suggesting that the markers of oxidative stress may be potentially predictive for progression of IgAN. Advanced oxidation protein products (AOPPs) are defined as dityrosine containing cross-linked protein products generated by monocyte activation and are recognized as the markers of oxidative stress. ${ }^{5}$ Our recent studies have demonstrated that chronic AOPP load in animals with or without CKD results in AOPP expression in renal tissue and promotes tissue inflammation and progression of renal fibrosis. ${ }^{6}$ Therefore, it is of clinical interest to test whether AOPPs are expressed in renal tissue in human IgAN and whether AOPP expression level or location predicts progression of renal fibrosis.

Therefore we conducted a prospective cohort study to test the hypothesis that renal AOPP expression level and location may predict disease progression of IgAN. In this cohort of 100 IgAN patients with normal renal function at presentation, we found that AOPPs accumulated in renal tissue and co-localized with pro-fibrotic factors transforming growth factor- $\beta 1$ (TGF- $\beta 1$ ) and angiotensin II (AngII). Renal AOPP expression intensity and localization closely correlated with the level of tissue cellular inflammation at the time of diagnosis and the higher staining score was associated with the risk of rapid progression of CKD during a 4-year followup. In 30 patients who received repeat renal biopsy at a mean of 42 months after diagnosis, renal AOPP expression at initial biopsy was independently predictive for progression of renal fibrosis even after adjustment with the known risk factors. This is the first study showing that renal AOPPs accumulation is an early predictor, detectable at the time of diagnosis, for renal progression in patients with relatively early stage of IgAN.

\section{MATERIALS AND METHODS Patients}

This is a single-center prospective cohort study approved by Guangdong Provincial Research Institute Review Board, and all subjects provided written consent. Between October 2005 and May 2010, patients who presented with biopsy-proven primary IgAN and normal renal function $\left(\mathrm{eGFR} \geq 80 \mathrm{ml} / \mathrm{min} / 1.73 \mathrm{~m}^{2}\right)$ were prospectively included. We included patients who presented with microhematuria, significant but non-nephrotic proteinuria, with or without hypertension, and normal renal function. This patient population represented the typical IgAN with low or moderate risk for disease progression at the time of diagnosis. ${ }^{7}$

Exclusion criteria include: those $<14$ or $>65$ years of age, those with cofounding diseases such as diabetes, liver disease, or infection, and those who carried a secondary renal pathological diagnosis. We also excluded patients who presented with eGFR $<80 \mathrm{ml} / \mathrm{min} / 1.73 \mathrm{~m}^{2}$ or with nephroticrange proteinuria ( $\geq 3.5 \mathrm{~g} / \mathrm{day})$ at the time of diagnosis. All the patients did not receive any treatment before diagnosis.

\section{Procedures}

This was an observational study. Treatment of IgAN was based on standard of $\operatorname{care}^{8}$ and entirely independent of the study. The treatment included prednisone plus renin angiotensin system (RAS) inhibitors $(n=67)$ or RAS inhibitors alone in patients who were unwilling to receive prednisone $(n=33)$. Blood samples and 24-h urine were collected before diagnostic biopsy and during follow-up. Seventy-seven (77\%) patients were followed at the outpatient clinic every 6-12 months for a mean of 4.2 years (3-7 years). Twentythree patients were lost after hospital discharge or during follow-up because of moving to other cities $(n=12)$ or lost connection $(n=11)$. Thirty $(30 \%)$ patients received repeat renal biopsy at a mean of 42 months (36-84 months) after diagnosis. The reasons for repeat biopsy include: evaluation of therapeutic response in patients with minor proteinuria or isolated hematuria at diagnosis $(n=14)$, to assess histological reversibility of $\operatorname{IgAN}(n=6)$, or to elucidate disease progression in patients who developed hypertension or persistent proteinuria that is resistant to therapy $(n=10)$.

\section{Outcome Definitions}

Rapid progression of CKD was defined as a sustained decline in eGFR of $>5 \mathrm{ml} / \mathrm{min} / 1.73 \mathrm{~m}^{2} /$ year. $^{9}$ Progression of renal fibrosis was defined as renal fibrosis index increased to $\geq 0.5$ in the repeat biopsy compared with that in the diagnostic biopsy.

\section{Renal Histological Evaluation}

Renal biopsy samples were reviewed and semi-quantitatively scored independently by two pathologists who were blinded to the identity of the tissue.

For analyzing glomerular sclerosis and tubulointerstitial fibrosis, the entire glomerular and interstitial compartments were scored separately using a semi-quantitative scoring system. ${ }^{10}$ Glomerular sclerosis was classified as the following criteria: 0 , no sclerosis; $1,<25 \%$ cross-sectional sclerosis; 2 , 25-50\% exhibiting sclerosis; and 3, >50\% cross-sectional sclerosis. Interstitial fibrosis was graded according to the following scale: 0 , no evidence of interstitial fibrosis; 1 , $<25 \%$ involvement; 2, 25-50\% involvement; and 3, > 50\% involvement. Oxford classification was applied as previously described. ${ }^{11}$ Vascular lesions, including arterial lesion and arteriolar hyaline, were scored using a semi-quantitative scoring system. ${ }^{11}$

\section{Renal Immunohistochemical Analyses}

AOPP expression was tested by using paraffin-embedded tissues sections cut at $5 \mu \mathrm{m}$. Renal tissue samples obtained from $100 \mathrm{IgAN}$ patients and 3 controls (normal kidney tissue adjacent to renal carcinoma obtained from nephrectomy). After de-paraffinization and blocking of endogeneous peroxide activity, slides were microwaved in high power for $2 \mathrm{~min}$ and in low power for $10 \mathrm{~min}$. The slides were then blocked with goat serum for $2 \mathrm{~h}$ and incubated with an 
anti-AOPPs $\mathrm{mAb}(3 \mathrm{~F} 2,1: 600)$ for $1 \mathrm{~h}$ at $37^{\circ} \mathrm{C}$. The primary anti-AOPPs $\mathrm{mAb}$ was produced by our laboratory and has been demonstrated to react specifically with hypochlorous acid-modified proteins but not with the native form or other oxidant-modified proteins. ${ }^{12}$ After washing with PBS, a secondary goat anti-mouse antibody was added for $30 \mathrm{~min}$ in room temperature. The slides were visualized by diaminobenzidine using the REAL EnVision Detection Kit (Dako, Glostrup, Denmark) following the manufacturer's instructions. Hematoxylin was applied as a counterstain.

Semi-quantitation of AOPP staining was performed by the method introduced by Xavier et al ${ }^{13}$ with minor modification. Briefly, the digital images of glomeruli or tubular interstitial area at $1360 \times 1024$ pixel resolution were captured at $\times 400$ magnification by the DP 71 CCD camera (Olympus, Japan) coupled to an Olympus AX-70 microscope (Olympus). We randomly selected the serial number of the captured digital areas for analysis by coin flipping. The staining score of AOPPs was calculated by the Image-Pro Plus software (version 6.0, Media Cybernetics, Silver Spring, MD) and expressed as the ratio of integrated optical density (IOD) to observed area (IOD/area), representing the relative expression level of AOPPs in glomeruli and tubulointerstitial area.

Renal expression of TGF- $\beta 1$, Ang II, and macrophage infiltration was assessed by the immunoperoxidase staining as described previously, ${ }^{6,14}$ using a rabbit anti-human TGF- $\beta 1$ polyclonal antibody (1:200, Santa Cruz Biotechnology, Santa Cruz, CA), a rabbit anti-human Ang II antibody (1:1500, Peninsula Laboratory, Los Gatos, CA), and an antihuman CD68 mAb (1:50, AbD Serotec, Kidlington, UK), separately.

The number of infiltrated macrophage was calculated by counting the CD68-positive cells in 10 randomly selected $0.25 \times 0.25 \mathrm{~mm}^{2}$ areas of tubulointerstitium. The staining scores of TGF- $\beta 1$ and Ang II were calculated as described above.

\section{Renal Immunofluorescence Assays}

Co-localization of AOPPs with TGF- $\beta 1$ or Ang II was determined by double staining of immunofluorescence in $5-\mu \mathrm{m}$ paraffin-embedded sections as previously described. ${ }^{14}$ Briefly, the slides were blocked with goat serum and then incubated with the mixture of an anti-AOPPs mAb $(1: 50,3 F 2)$ and an anti-TGF- $\beta 1$ antibody (1:100, Santa Cruz) or an anti-AOPPs mAb (1:50, 3F2) and an anti-Ang II antibody (1:200, Peninsula Laboratory) overnight at $4{ }^{\circ} \mathrm{C}$. The immunofluorescence staining was performed using dylight 488 conjugated goat anti-rabbit antibody (1:50, Jackson ImmunoResearch Laboratories, West Grove, PA) or dylight 594 conjugated goat anti-mouse antibody (1:100, Jackson ImmunoResearch Laboratories) at $37^{\circ} \mathrm{C}$ for $2 \mathrm{~h}$.

\section{Laboratory Measurements}

Plasma AOPP levels were determined at the time of diagnosis in all patients using a modified spectrophotometry method previously devised. ${ }^{15}$ Serum levels of creatinine, lipids, C-reactive protein, and IgA were detected by Olympus AU5400 auto-analyzer (Olympus, Japan). The eGFR was calculated using the CKD-EPI formula. ${ }^{16}$ Urinary protein excretion was measured by the biuret method using the $24-\mathrm{h}$ collected urine samples.

\section{Statistical Analyses}

The sample size estimation was conducted with the nQuery Adivsor software 7.0 (Statistical Solutions Ltd., Cork, Ireland). Because no data on predictive effect of renal AOPP expression in patients with IgAN were available, we assumed that the predictive accuracy should be at least $70 \%$ to meet clinical implication. We expected that using renal AOPP expression as a marker would yield a diagnostic accuracy of $84 \%$. The sample size was estimated at 75 cases by setting a statistical power of $80 \%$ at a two-sided significance level of 0.05 .

Statistical analysis was performed using the SPSS 17.0 (SPSS, Chicago, IL). The continuous variables were expressed as mean \pm s.d. or median (interquartile range). Categorical variables were expressed as percentages. The Student's $t$-test and the Mann-Whitney $U$ test were used to compare normally and not normally distributed variables, respectively. The categorical data were compared with Pearson $\chi^{2}$ test. Correlations were determined using the Pearson's correlation coefficient for normally distributed data and the Spearman's rank correlation coefficient for non-normally distributed data. Multiple linear regression analysis was conducted to determine the variables associated with the staining score of AOPPs. The independent effects of variables on the staining scores of AOPP were analyzed by stepwise multivariate linear regression analysis $(P<0.05$ for entry and $P \geq 0.05$ for removal). To analyze the predictors for progression of renal fibrosis or rapid progression of CKD, multivariable logistic regression analysis was performed. All $P$ values were twotailed and those $<0.05$ were considered significant. To determine the performance of renal AOPP expression for predicting rapid progression of CKD or progression of renal fibrosis, a conventional receiver-operating characteristic curve was generated and the area under the curve (AUC) of renal AOPP expression was analyzed.

\section{RESULTS \\ AOPP Expression in Renal Tissue from Patients with IgAN}

Patients' characteristics at the time of diagnosis are shown in Table 1. Included patients presented relatively low-grade tissue lesions.

As shown in Figure 1a, the expression of AOPPs was detected predominantly in podocytes and mesangial cells (a1), arterial/arteriolar endothelial cells (a2), interstitialinfiltrating cells (a3 and a4), and tubule epithelial cells (a5). The specificity of the immunoreactivity was confirmed by the positive staining using an anti-AOPPs mAb preincubated 
Table 1 Characteristics of patients with IgA nephropathy at diagnosis $^{\mathrm{a}}$

$\begin{array}{ll}\text { Variables at the time of diagnosis } & \text { Data } \\ \text { Clinical information } & \\ \text { Age, years } & 31.8 \pm 9.7 \\ \text { Male, } n(\%) & 42(42) \\ \text { BMl, } \mathrm{kg} / \mathrm{m}^{2} & 22.31 \pm 3.36 \\ \text { Hypertension, } n(\%) & 26(26) \\ \text { Systolic blood pressure, } \mathrm{mm} \mathrm{Hg} & 128.4 \pm 19.3 \\ \text { Diastolic blood pressure, } \mathrm{mm} \mathrm{Hg} & 80.5 \pm 13.0 \\ \text { eGFR, } \mathrm{ml} / \mathrm{min} / 1.73 \mathrm{~m}^{2} \mathrm{~b} & 103.6 \pm 24.0\end{array}$

Serum measurement

Albumin, g/l

$39.52 \pm 5.20$

Low-density lipoprotein cholesterol, mmol//

$2.47 \pm 0.76$

High-density lipoprotein cholesterol, mmol/l

$1.50 \pm 0.51$

$\lg \mathrm{A}, \mathrm{g} / \mathrm{l}$

$2.97 \pm 1.03$

Creatinine, $\mu \mathrm{mol} / \mathrm{l}$

C-reactive protein, $\mathrm{mg} / \mathrm{l}$

$71.03 \pm 16.65$

$1.10 \pm 0.98$

Advanced oxidative protein products, $\mu \mathrm{mol} / \mathrm{l}$

$58.30 \pm 11.87$

Urine measurement

Proteinuria, g/day

$0.86(0.45-1.41)$

Macrohematuria, $n$ (\%)

$20(20)$

Microscopic hematuria, $n(\%)$

$60(60)$

Oxford-MEST grade

Mesangialhypercellularity (M1), $n$ (\%)

$80(80)$

Endocapillaryhypercellularity (E1), n (\%)

$38(38)$

Segmental glomerulosclerosis (S1), $n$ (\%)

$68(68)$

Tubular atrophy/interstitial fibrosis

TO, $n(\%)$

$61(61)$

$\mathrm{T} 1, n(\%)$

39 (39)

Arterial lesions index

$0.36 \pm 0.50$

Arteriolar hyaline index

$0.16 \pm 0.40$

Renal fibrosis index

Glomerular sclerosis index

$0.37(0.13-0.70)$

Tubulointerstitial fibrosis index

$1.00(0.70-1.30)$

${ }^{\mathrm{a}} N=100$, continuous variables are expressed as mean \pm s.d. or median (25th percentile-75th percentile). Categorical variables are expressed as number (percentage).

beGFR was determined using CKD-EPI formula.

with native albumin (a6) and the negative staining using an anti-AOPPs $\mathrm{mAb}$ preincubated with AOPPs-albumin (a7) or non-immune IgG instead of primary mAb (a8). In most of the patients, renal AOPP expression was evenly distributed in renal cortex. As shown in Figure 1b, all IgAN patients in this cohort had AOPP expression in glomeruli and tubules. AOPP expression was found in arterial endothelial cells in $88 \%$ of the patients and in interstitial infiltration cells in $27 \%$ of the patients. The staining score of AOPPs was higher in tubular interstitial area than in glomeruli $(P<0.01$, Figure 1c). The normal kidney tissue adjacent to renal carcinoma obtained from nephrectomy was used as the normal controls in the immunohistochemical analysis. There was no AOPP expression in the normal renal tissues (Figure 2a).

\section{Renal AOPP expression Closely Correlated with Tissue Inflammation}

To determine the factors that correlate with the renal AOPP expression, we performed a Spearman's rank correlation test to estimate relationships between the variables. Multiple linear regression analysis was then performed to determine the independent relationship of the variables with renal AOPPs. As shown in Table 2, the number of glomerular macrophage and mesangial hypercellularity were significantly correlated with glomerular AOPP expression. Twenty-six percent $\left(R^{2}\right)$ of the variance of glomerular AOPP expression could be predicted by the two parameters. In addition, plasma AOPP level and the number of interstitial macrophage were significantly correlated with AOPP staining score in tubulointerstitial area. Forty-one percent of the variance of tubulointerstitial AOPPs could be predicted by these parameters. Renal AOPP expression also correlated with histological lesions scored by MEST (Supplementary Table S1).

Because plasma AOPP levels were independently associated with AOPP expression in tubulointerstitial area, we further compared the level of interstitial macrophage infiltration in patients stratified by the level of plasma AOPPs (greater or less than or equal to the mean). Interstitial macrophage infiltration was more obvious in patients with higher tubulointerstitial AOPPs score (greater than the median) than those with lower expression (less than or equal to the median) in each category of plasma AOPPs (Supplementary Figure S1).

\section{Correlation between Location of Renal AOPPs and Clinical Manifestations at the Time of Diagnosis}

As AOPP expression was found in interstitial-infiltrating cells and arterial/arteriolar endothelium in a portion of patients with IgAN, we next compared the major clinical and histological variables between the patients with or without AOPP expression in these sites. Compared with those with negative AOPP expression in interstitial-infiltrating cells, patients with positive AOPP expression showed increased prevalence of hypertension (15.5 vs $48.3 \%, P=0.001)$ and higher tubulointerstitial lesion grades of Oxford-MEST (28.2 vs 62.1\%, $P=0.002)$. Interestingly, patients with positive staining of AOPPs in vascular endothelial cells had a significant increase 

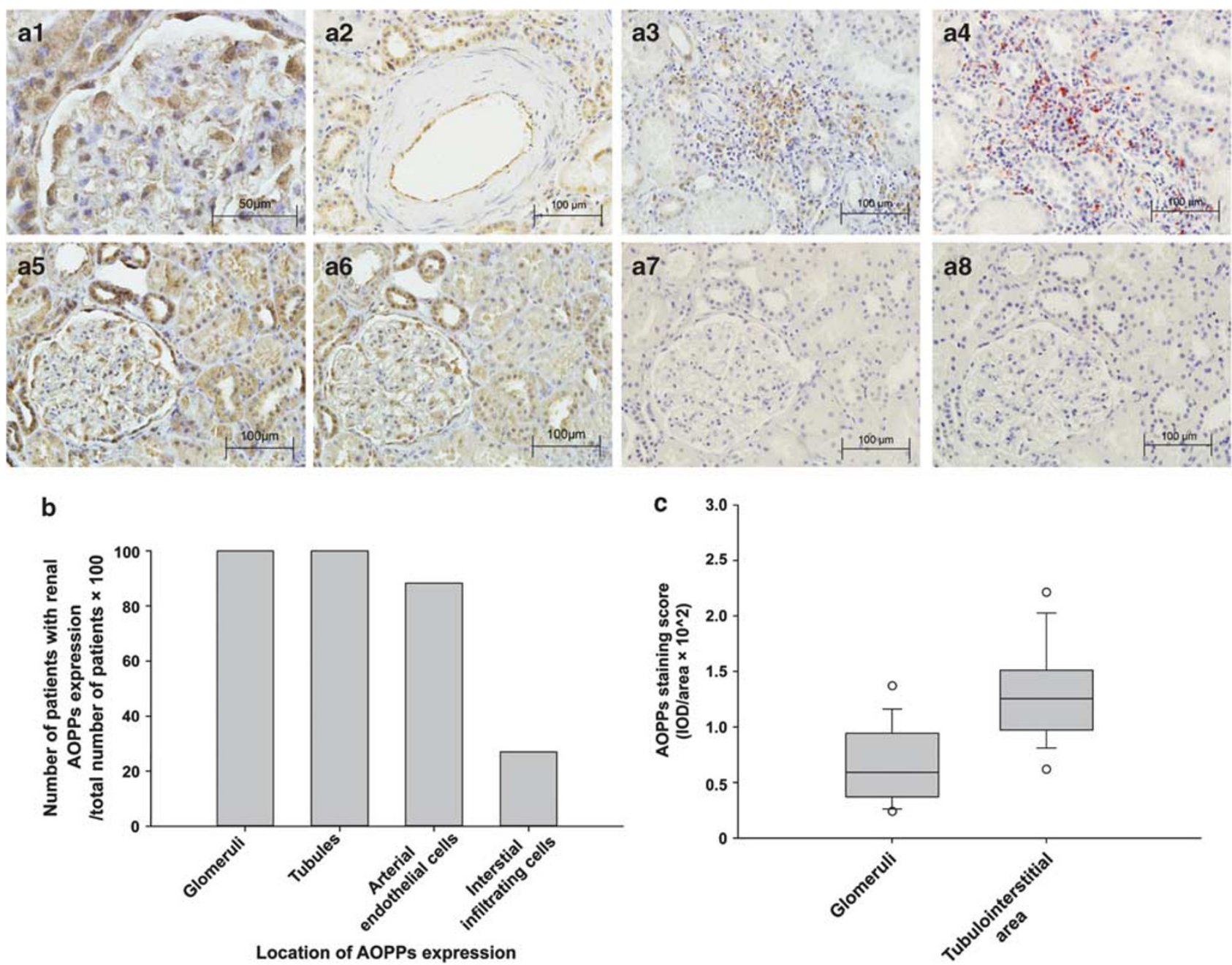

Figure 1. AOPP expression and distribution in renal tissues obtained from patients with IgAN. (a1-a4) A representative photo of AOPP distribution in renal tissues from a patient with IgAN. Expression of AOPPs was detected in glomeruli (a1), tubular epithelial cells (a2), arterial endothelial cells (a3), and the interstitial-infiltrating cells (a4). Anti-CD68 staining showed that AOPP-positive cells were mainly macrophages (a4). (a5-a8) Specificity of AOPP staining in the sequential sections from a patient with IgAN. The sections were stained using an anti-AOPP mAb (a5), an anti-AOPP mAb preincubated with human serum albumin (a6), an anti-AOPP mAb preincubated with AOPP-albumin (a7), or a homotype control of mouse IgG (a8), separately. (b) All IgAN patients in the cohort had AOPP expression in glomeruli and tubules. AOPP expression was found in arterial endothelial cells in $88 \%$ of the patients and in interstitial infiltration cells in $27 \%$ of the patients. (c) Semi-quantitative analysis of AOPP staining score in glomeruli and tubulointerstitial area. The horizontal line is the median, the edges of the box represent the 25th and 75th percentiles, the bars represent values within 5th and 95th percentiles, and outliers $(O)$ are shown.

in arterial lesion score compared with those with negative AOPP staining. Twenty-eight percent (25/87) of patients with AOPP expression in artery/arteriole were hypertensive compared with zero in those with negative staining of AOPPs in endothelium $(0 / 13)$.

\section{Renal AOPPs was Co-Localized with Profibrotic Factors}

Overexpression of TGF- $\beta 1$ and activation of local RAS have been demonstrated to have a critical role in progression of renal fibrosis. ${ }^{17,18}$ To investigate the relationship between renal AOPPs and these profibrotic factors, we performed immunohistological studies in serial renal sections from patients with IgAN (Figure 2a). AOPP expression co-loca- lized with TGF- $\beta 1$ and Ang II, particularly in tubule epithelial cells. Double staining of AOPPs with anti-TGF- $\beta 1$ or anti-Ang II showed similar pattern (Figure 2b). The staining scores of AOPPs in tubulointerstitial area were positively correlated with the staining scores of TGF- $\beta 1$ and Ang II expression at diagnosis (Figure 2c).

\section{Renal AOPP expression at Initial Biopsy Predicted Rapid Progression of CKD during Follow-up}

Seventy-seven $(77 \%)$ patients in the cohort were followed for a mean of 4.2 years. Among these patients, 25 (32\%) were treated with RAS inhibitors and 52 were $(68 \%)$ treated with a combination of steroid and RAS inhibitors. There was no 
Rabbit IgG
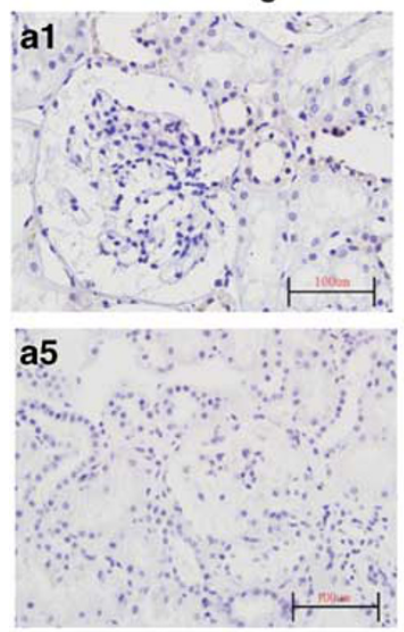

AOPPS

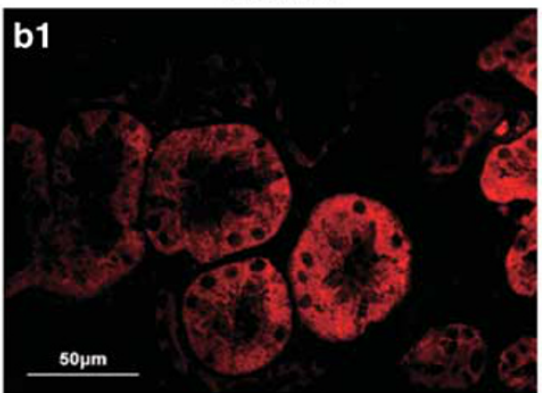

AOPPs
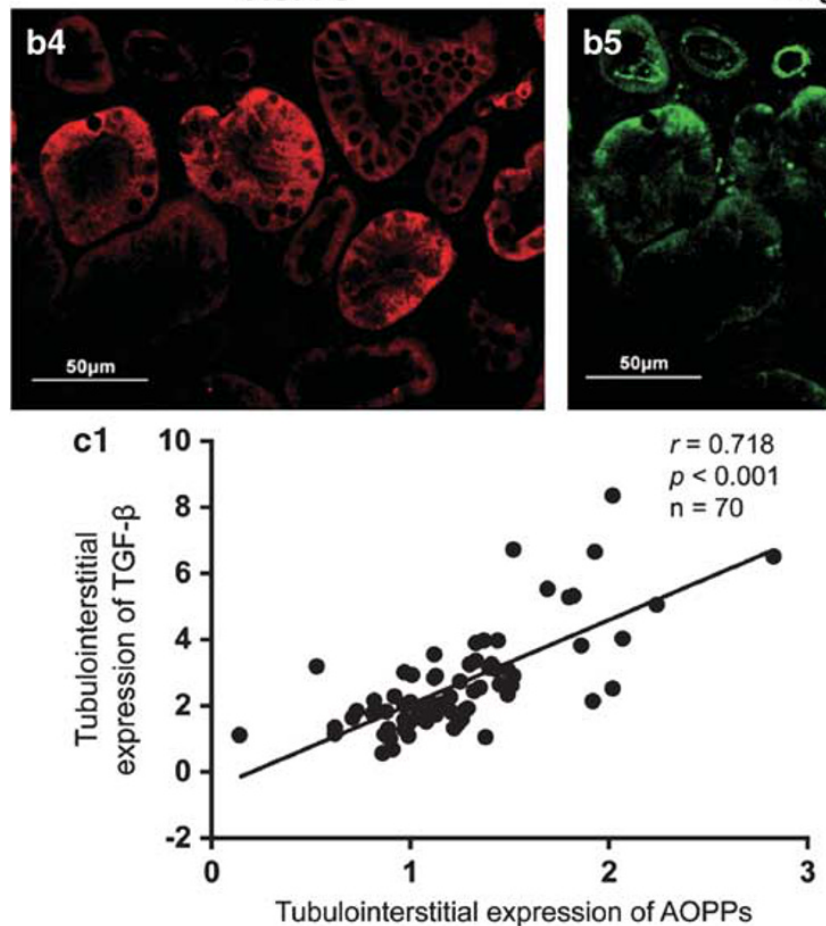

AOPPs

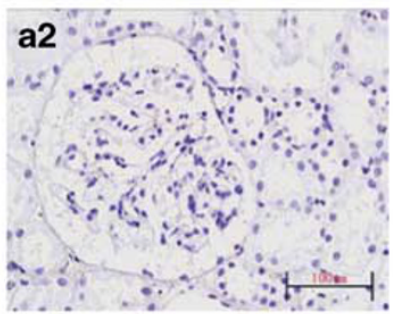

a6

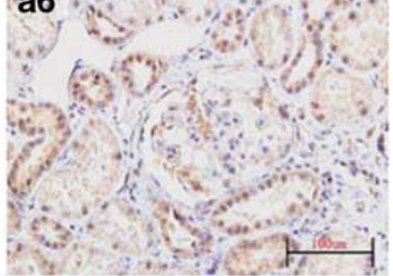

TGF- $\beta$

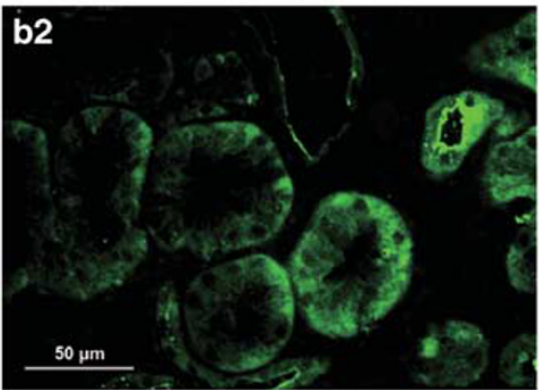

Angll

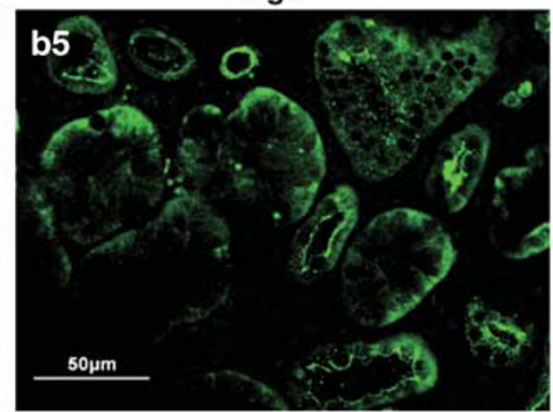

c2
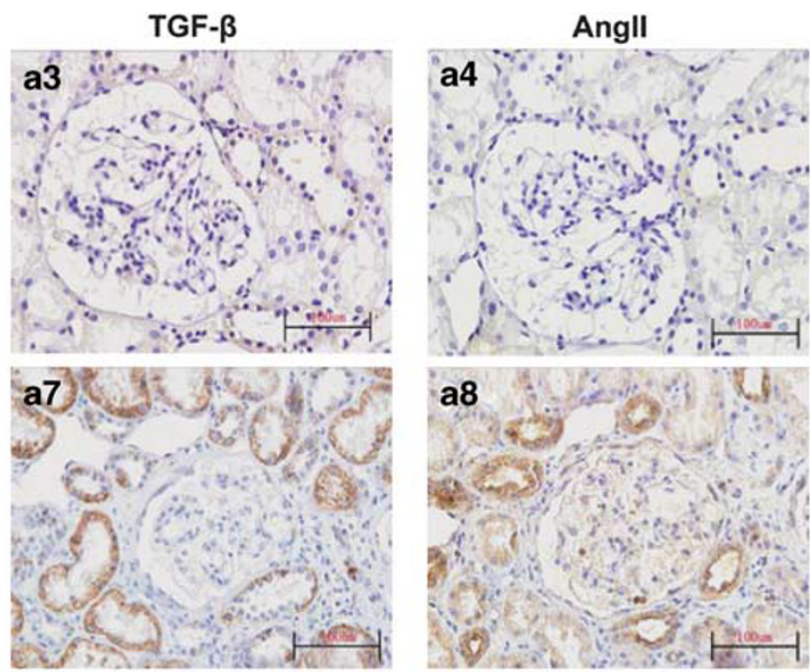

Merge

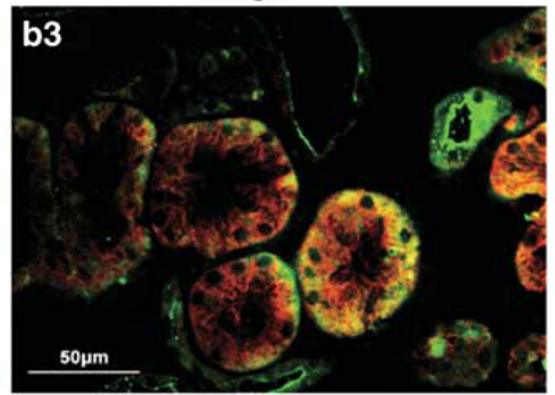

Merge
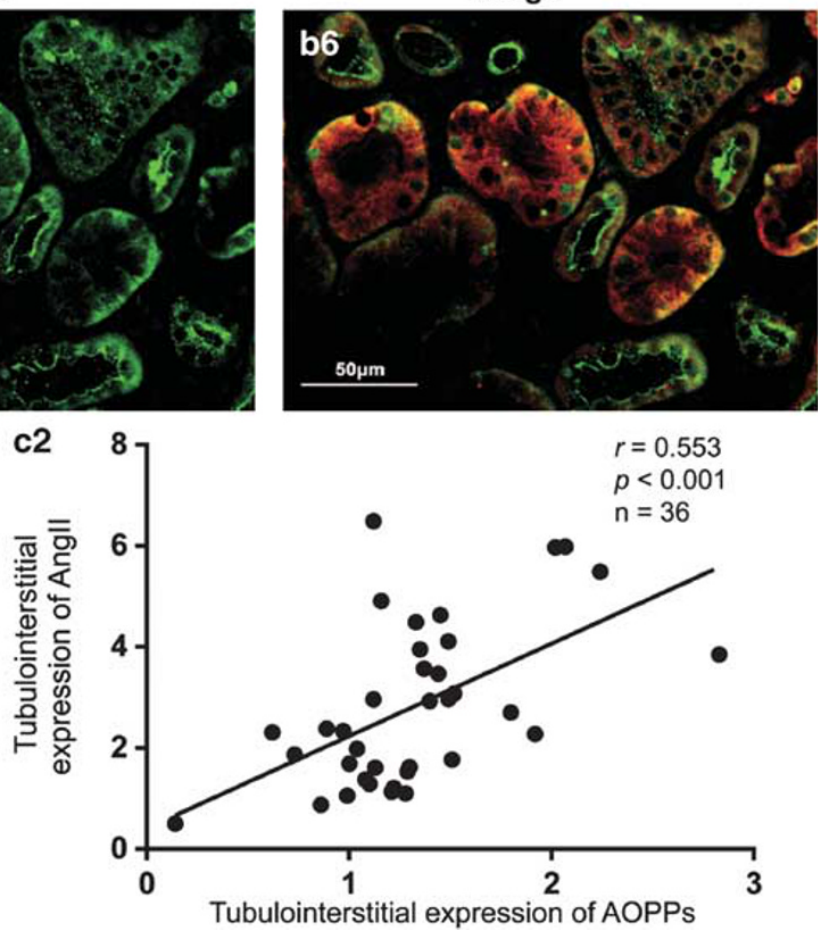

Figure 2 Expression of AOPP, TGF- $\beta 1$, and angiotensin II in the renal tissue obtained from patients with IgAN (a) A representative immunohistochemical staining of serial sections from a normal control (a1-a4) and a patient with IgAN (a5-a8) using a non-immune rabbit IgG (a1, a5), an anti-AOPP mAb (A2, A6), an anti-TGF- $\beta 1$ antibody (a3, a6), or an anti-angiotensin II antibody (a4, a8), separately. (b) A representative photo of immunochemical double-staining in renal tissue from a patient with IgAN, showing that AOPP expression was co-localized with TGF- $\beta 1$ (b1-b3) and angiotensin II (b4-b6). (c) The correlation of renal AOPPs with TGF- $\beta 1$ (c1) and angiotensin II expression (c2) in the tubulointerstitial area. Scale bars: $100 \mu \mathrm{m}$. 
Table 2. Multivariate linear regression: variables correlated with renal AOPP expression at diagnosis ${ }^{a}$

\begin{tabular}{llrl}
\hline Dependent variables & Independent variables & $\beta^{\text {b }}$ & $P$ \\
Glomerular staining score of AOPPs & Number of glomerular macrophage & 0.230 & 0.003 \\
& Mesangialhypercellularity & 0.187 & 0.023 \\
Tubulointerstitial staining score of AOPPs & Plasma AOPP level & 0.347 & $<.001$ \\
& Number of interstitial macrophage & 0.348 & $<0.001$ \\
\end{tabular}

${ }^{\mathrm{a}} \mathrm{N}=100$, variables entered the univariate analysis, including age, BMI, blood pressure, serum cholesterol, serum lgA, serum creatinine, CRP, proteinuria, renal macrophage infiltration, and the histological lesions (Oxford-MEST). The parameters with $P<0.05$ in the univariate analysis entered the multivariate regression analysis. Only parameters with $P<0.05$ have been shown in the table.

$\mathrm{b} \beta$ is the standard coefficient.

a1

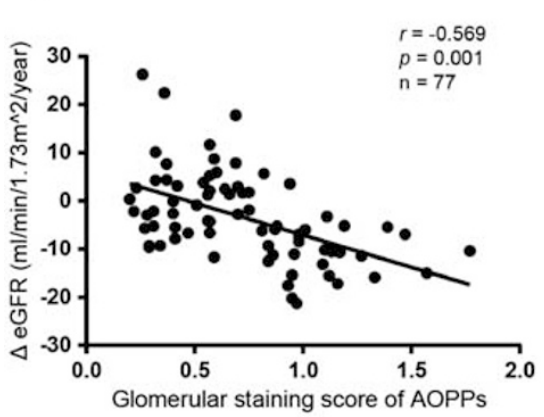

b1

$\square$ Glomerular AOPPS staining score $\leq 0.64$
Glomerular AOPPs staining score $>0.64$

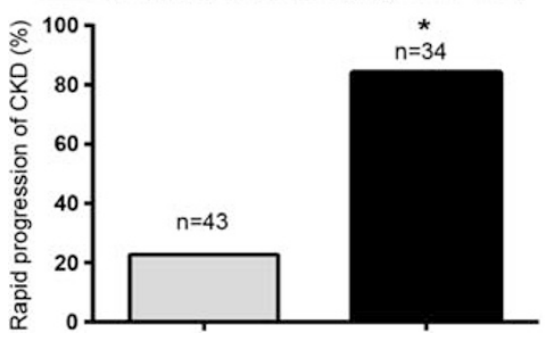

a2

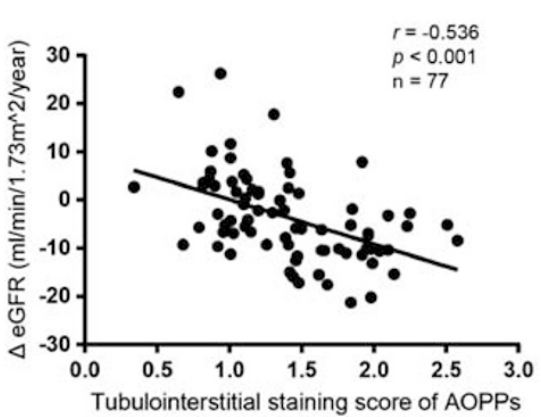

b2

Tubulointerstitial AOPPS staining score $\leq 1.29$

Tubulointerstitial AOPPs staining score $>1.29$

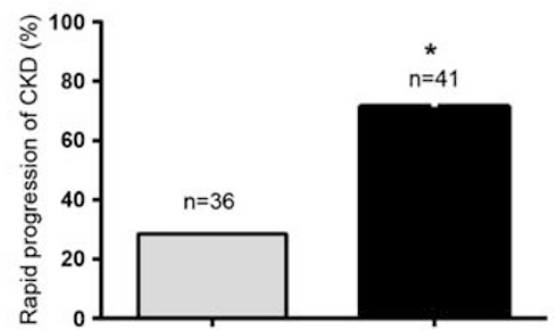

a3 $\square$ AOPPs positive in interstitial infiltrating cells aOPPs negative in interstitial infiltrating cells

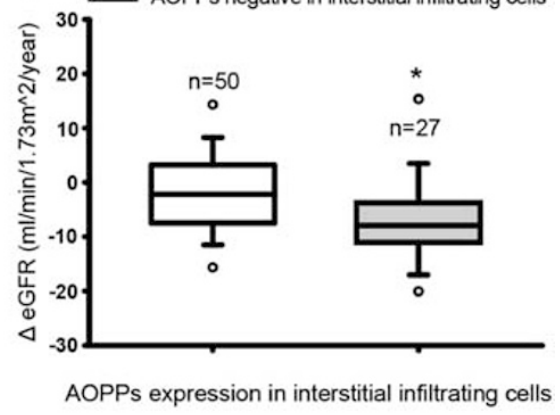

b3

\section{$\square$ AOPPs negative in interstitial infiltrating cells}

AOPPs positive in interstitial infiltrating cells

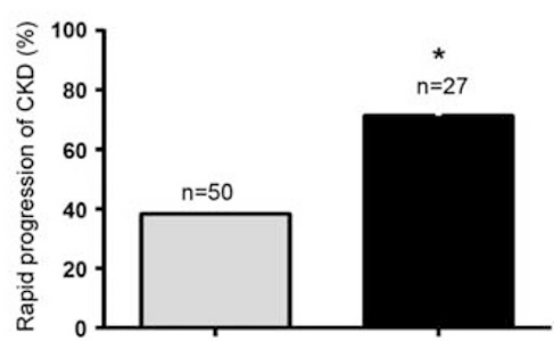

Figure 3. Renal AOPP expression at diagnosis was associated with rapid progression of CKD during follow-up. (a) The relationships between renal AOPP expression at diagnosis and the changes $(\Delta)$ of eGFR during follow-up in 77 patients with lgAN (a1-a3). (b) Renal AOPP staining score greater than the median (b1 and b2) or positive AOPPs in interstial-infiltrating cells (b3) was associated with significantly higher incidence of rapid progression of CKD as compared with AOPP expression less than or equal to the median or negative AOPPs in interstitial-infiltrating cells. ${ }^{*}<0.05$.

statistically significant difference in AOPP expression and MEST score between patients who did and did not subsequently receive steroid therapy (Supplementary Table S2). No statistically significant difference was found in the proportion of patients with rapid progression of CKD between those treated with (27/52) and without (13/25) steroid therapy $(P>0.05)$.

As shown in Figure 3a, glomerular or tubulointerstitial staining score of AOPPs at diagnosis was negatively correlated with the changes of eGFR during follow-up. Compared with those with negative AOPP staining, patients with positive staining of AOPPs in interstitial-infiltrating cells had more obvious decline of eGFR during follow-up. Renal AOPP staining score greater than the median at diagnosis was associated with higher incidence of rapid progression of CKD with to those with score less than or equal to the median (Figure 3b). MEST criteria also had trend for predicting changes of proteinuria and eGFR, but only glomerular 
sclerosis (S1) reached statistical significance (Supplementary Table S3).

To examine the predictive performance of renal AOPP expression on rapid progression of CKD, the AUC of renal AOPP staining scores at different cutoff values were generated. Patients with renal AOPP staining scores greater than the best cutoff at diagnosis had higher incidence of rapid progression of CKD compared with those with the staining scores less than or equal to the best cutoff (Supplementary Figure S2).
Renal AOPP staining score higher than the best cutoff or AOPP positive expression in interstitial-infiltrating cells independently predicted rapid progression of CKD in a multivariable logistic regression analysis even adjusted for histological changes (MEST) at baseline, renal inflammation, and other known clinical risk factors (Table 3). The risk of renal function decline $\left(>5 \mathrm{ml} / \mathrm{min} / 1.73 \mathrm{~m}^{2} /\right.$ year $)$ in patients treated with steroid appeared to be similar among subgroups classified according to glomerular AOPP levels and histological lesions (M1 and E1) (Supplementary Table S4).

Table 3 Multivariate logistic regression analysis ${ }^{a}$ : prediction of renal AOPPs for rapid progression of CKD

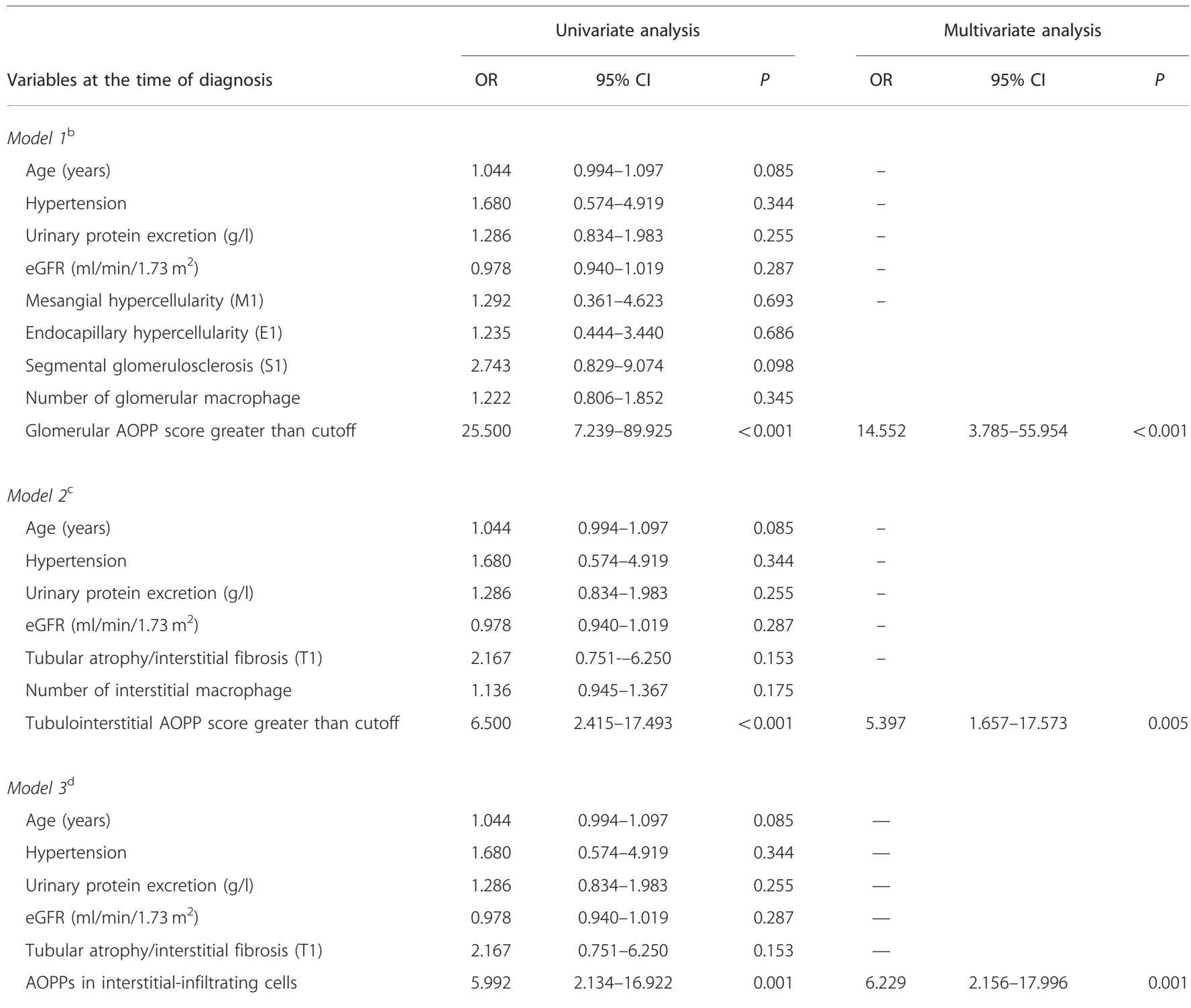

Abbreviations: $\mathrm{OR}$, odds ratio; $\mathrm{Cl}$, confidence interval.

${ }^{\mathrm{a}} \mathrm{N}=77$, rapid progression of CKD was defined as a sustained decline in eGFR of $>5 \mathrm{ml} / \mathrm{min} / 1.73 \mathrm{~m}^{2} / \mathrm{year}$.

${ }^{b}$ Prediction of glomerular AOPPs for progression of glomerular sclerosis.

'Prediction of tubulointerstitial AOPPs for progression of tubulointerstitial fibrosis.

${ }^{\mathrm{d}}$ Prediction of AOPPs in interstitial-infiltrating cells for progression of tubulointerstitial fibrosis. 
However, there is no statistically significant difference in the progression of CKD between patients with baseline plasma AOPP level higher and lower than the median $(58.3 \mu \mathrm{mol} / \mathrm{l})$ (data not shown).

\section{Renal AOPP expression at Initial Biopsy Predicted Progression of Renal Fibrosis}

Thirty patients received repeat biopsy at a mean of 42 months. The characteristics of these patients at the time of diagnosis and repeat biopsy are shown in Table 4. Urinary protein excretion rate was significantly reduced at the time of repeat biopsy, while tubulointerstitial lesion (Oxford-MEST) and renal fibrosis score significantly increased. There were no significant changes in blood pressure and the level of serum creatinine. No statistically significant difference was found in the changes of macrophage infiltration and MEST between patients treated with (20/30) and without (10/30) steroid therapy (Supplementary Table S5).

As shown in Figure 4a, renal AOPP expression at initial biopsy was positively correlated with changes of renal fibrosis score at repeat biopsy. A staining score of AOPPs

Table 4 Characteristics of IgAN patients who received repeat renal biopsy ${ }^{a}$

\begin{tabular}{|c|c|c|c|}
\hline & $\begin{array}{l}\text { On time of } \\
\text { diagnosis }\end{array}$ & $\begin{array}{l}\text { On time of } \\
\text { repeat biopsy }\end{array}$ & $P$ \\
\hline \multicolumn{4}{|l|}{ Clinical information } \\
\hline Age, years & $30.2 \pm 10.5$ & $33.5 \pm 11.0$ & - \\
\hline Systolic blood pressure, mm Hg & $123.9 \pm 14.7$ & $122.3 \pm 15.0$ & 0.672 \\
\hline Diastolic blood pressure, $\mathrm{mm} \mathrm{Hg}$ & $78.0 \pm 12.9$ & $76.1 \pm 11.0$ & 0.391 \\
\hline Serum creatinine, $\mu \mathrm{mol} / / \mathrm{l}$ & $73.5 \pm 12.4$ & $79.1 \pm 25.1$ & 0.231 \\
\hline Urine protein excretion, g/day & $1.12(0.67-1.86)$ & $0.37(0.16-2.10)$ & 0.021 \\
\hline eGFR, $\mathrm{ml} / \mathrm{min} / 1.73 \mathrm{~m}^{2}$ & $100.7 \pm 21.0$ & $95.5 \pm 29.7$ & 0.361 \\
\hline \multicolumn{4}{|l|}{ Oxford-MEST } \\
\hline $\mathrm{M} 1, n(\%)$ & $22(73.3 \%)$ & $20(66.7 \%)$ & 0.541 \\
\hline $\mathrm{E} 1, n(\%)$ & $9(30.0 \%)$ & $7(23.3 \%)$ & 0.597 \\
\hline$S 1, n(\%)$ & $19(63.3 \%)$ & $23(76.7 \%)$ & 0.221 \\
\hline T0, $n(\%)$ & $23(76.7 \%)$ & $13(43.3 \%)$ & 0.016 \\
\hline $\mathrm{T} 1, n(\%)$ & 7 (23.3\%) & $13(43.3 \%)$ & \\
\hline $\mathrm{T} 2, n(\%)$ & 0 & $4(7.5 \%)$ & \\
\hline
\end{tabular}

Renal fibrosis score

Glomerular sclerosis index

$0.33(0.06-0.60) \quad 0.67(0.36-1.19) \quad 0.003$

Tubulointerstitial fibrosis index

$0.85(0.60-1.08) \quad 1.45(0.63-1.80) \quad 0.009$

${ }^{\mathrm{a}} N=30$, continuous variables are expressed as mean $\pm \mathrm{s.d}$. or median (25th percentile-75th percentile). Categorical variables are expressed as number (percentage). greater than the median or positive staining of AOPPs in interstitial-infiltrating cells at diagnosis was associated with an increased risk of renal fibrosis score at the time of repeat biopsy (Figure 4b). Renal AOPP staining scores greater than the best cutoff at baseline was also associated with higher incidence of renal fibrosis progression compared with those with the staining scores less than or equal to the best cutoff (Supplementary Figure S2).

Renal AOPP staining score higher than the best cutoff and AOPP positive expression in interstitial-infiltrating cells were the independent predictors for progression of renal fibrosis in a multivariate logistic regression analysis even adjusted for histological changes (MEST) at baseline, renal inflammation, and other known clinical risk factors (Table 5).

\section{DISCUSSION}

The clinical outcome of patients with IgAN is highly variable. The limited ability to predict outcome in individual IgAN patients emphasizes the need to identify new markers for disease progression. ${ }^{19}$ The present study demonstrated that renal AOPP expression level and distribution were associated with tissue cellular inflammation in IgAN patients presented with relatively low-grade lesions and normal renal function. Higher level of renal AOPP expression at diagnosis independently predicted the risk of rapid progression of CKD and progression of renal fibrosis during follow-up. To the best of our knowledge, this is the first report indicating that renal AOPP expression might be an early predictor, detectable at the time of diagnosis, for renal progression in patients with relatively early stage of IgAN.

Although there is a general consensus regarding easily measurable clinical risk factors associated with adverse prognosis such as proteinuria, ${ }^{20}$ not all patients with 'lowrisk' clinical features have a benign course. ${ }^{21}$ Glomerulosclerosis and interstitial fibrosis are strong independent predictors of progression, ${ }^{1}$ but these histological changes are advanced lesions and had limited predictive value in patients with early disease. Therefore, it would be ideal to have early indicators to identify patients at high risk of progressive disease. The present study provides several lines of evidence supporting that AOPP accumulation in renal tissue might be a useful predictor for renal progression in patients with relatively early stage of IgAN. First, overexpression of AOPPs in glomeruli and tubulointerstitial area closely correlated with tissue cellular inflammation such as mesangial hypercellularity and macrophage infiltration in patients who met the characteristics of early or low-grade lesions of IgAN. ${ }^{22}$ Renal AOPP expression may be not specific for IgAN, as it can also be found in other CKD, such as diabetic nephropathy. ${ }^{12}$ Co-localization of AOPPs with TGF$\beta 1$ and Ang II supports that AOPP expression are associated with pro-inflammatory and pro-fibrotic processes in the kidney. Irrespective of the initial injury to the tissue, inflammation is closely related to tissue scar formation. ${ }^{23}$ Second, the level of renal AOPP expression at initial biopsy 

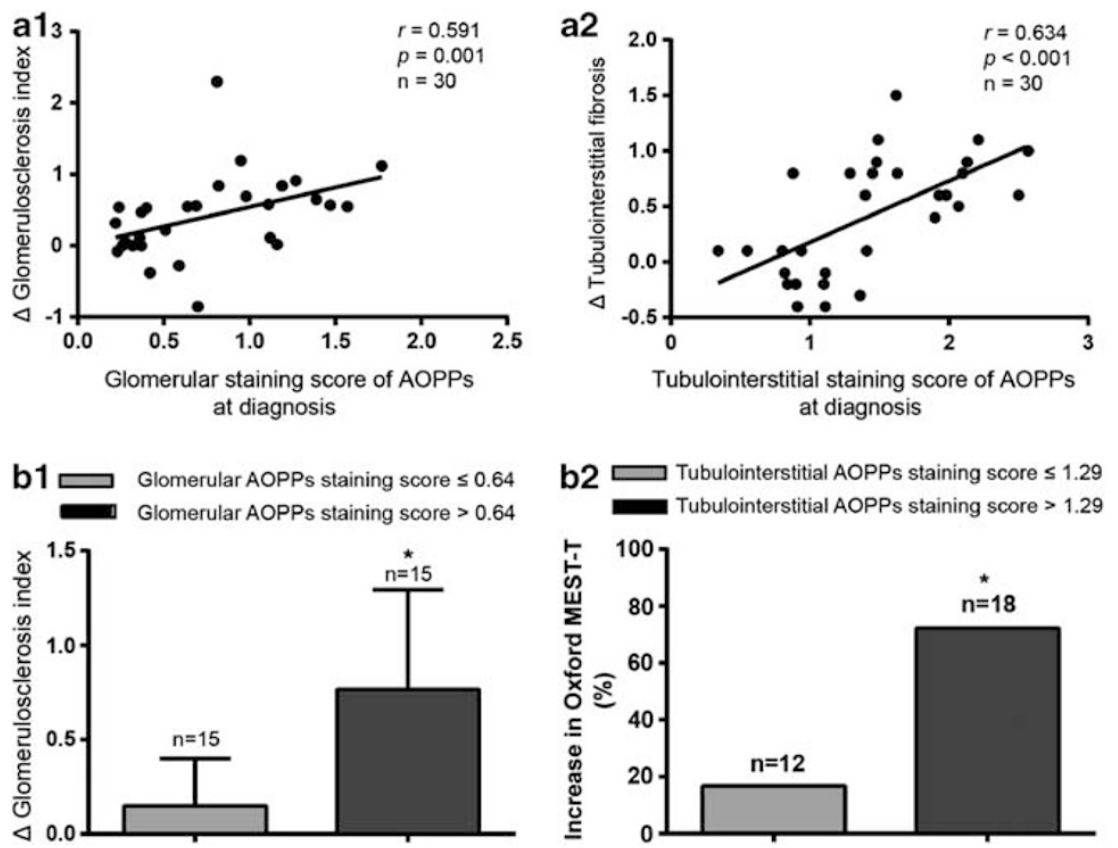

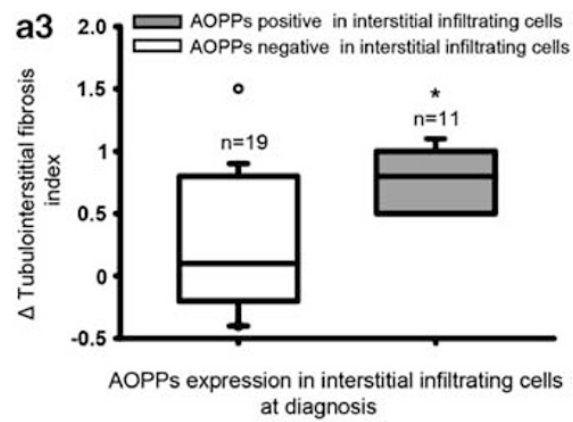

b3 Tubulointerstitial AOPPs staining score $\leq 1.29$ Tubulointerstitial AOPPs staining score $>1.29$

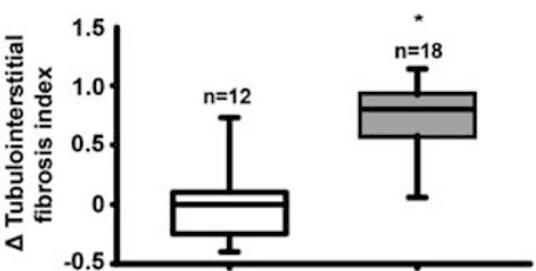

Figure 4. Renal AOPP expression at diagnosis was associated with progression of renal fibrosis in patients with IgAN (a) The relationships between renal AOPP staining scores at diagnosis and the changes $(\Delta)$ of renal fibrosis index at repeat biopsy in 30 patients with IgAN (a1-a3). (b) Renal AOPP expression greater than the median at diagnosis was associated with increase in glomerular sclerosis index (b1), Oxford-MEST-T grade (b2), and tubularinterstitial fibrosis index (b3) compared with AOPP expression less than or equal to the median. ${ }^{*} P<0.05$.

closely correlated with increase in renal fibrosis index in 30 IgAN patients who received repeat renal biopsy. Renal AOPP expression was also associated with rapid progression of CKD during follow-up. Higher level of renal AOPP expression at initial biopsy was associated with significantly increased likelihood of renal fibrosis progression and rapid progression of CKD in multivariate regression analysis adjusted for other known risk factors. Third, patients who had AOPP expression in interstitial-infiltrating cells showed more prevalence of hypertension and more severe renal histological injury. AOPP localization in interstitial-infiltrating cells was an independent predictor for progression of renal fibrosis even after multivariate adjustment. Given that renal fibrosis is the common pathway for progression of $\mathrm{CKD},{ }^{24}$ our results suggest that renal AOPP expression might be a novel marker for risk stratification in patients with IgAN.

Our results support the previous reports in which circulating AOPP level is predictive for renal surviving in patients with IgAN. ${ }^{25}$ Plasma AOPPs may be generated by activated monocytes during oxidative stress seen in patients with IgAN. ${ }^{5}$ Although our data failed to find the difference in progression of IgAN between those with baseline plasma AOPP level higher and lower than the median, we could not exclude the possibility that this may relate to the characteristic of the cohort which included only those with low or moderate risk for renal progression. Furthermore, measurement of plasma AOPPs has been questioned, because it is interfered by the confounding factors, such as hyperlipidemia. ${ }^{26}$ Besides reflecting progressive disease, higher plasma AOPPs concentration can also result from impaired renal function. ${ }^{27}$ These factors may limit the predictive value of circulating AOPPs in a portion of IgAN patients. The finding that renal AOPP accumulation reflected progression of renal fibrosis supports the hypothesis that the oxidative milieu is a risk factor for progression of IgAN. In addition to being markers of oxidative protein damage, AOPPs have an important role as effector molecules, by activating monocytes and triggering respiratory burst and inflammation, ${ }^{28}$ and inducing vascular endothelial dysfunction by activating endothelial cells. ${ }^{29}$ AOPPs, through triggering intracellular production of superoxide, induce podocyte depletion, ${ }^{30}$ increase synthesis of extracellular matrix in mesangial cells, ${ }^{31}$ and activate RAS in tubular epithelial cells. ${ }^{14}$ The clinical support for this hypothesis would be that antioxidant therapy is effective in treating CKD, including IgAN. ${ }^{32,33}$ Given that oxidative stress and inflammation occur early and might be modifiable in IgAN, ${ }^{5}$ therapeutic strategies aimed at reducing oxidative stress and inflammation seem to be very promising for future intervention of IgAN.

Up to now, our ability to reliably risk-stratify patients with IgAN at the time of diagnosis or during early follow-up remains limited. In most published studies, multivariate analysis based on renal survivorship identified impaired renal function and heavy proteinuria at presentation as the main predictors of poor outcome in adult patients. ${ }^{34-36}$ Urinary protein excretion in excess of $2 \mathrm{~g}$ /day is a strong marker of a 
Table 5 Multivariate logistic regression analysis ${ }^{a}$ : prediction of renal AOPPs for progression of renal fibrosis

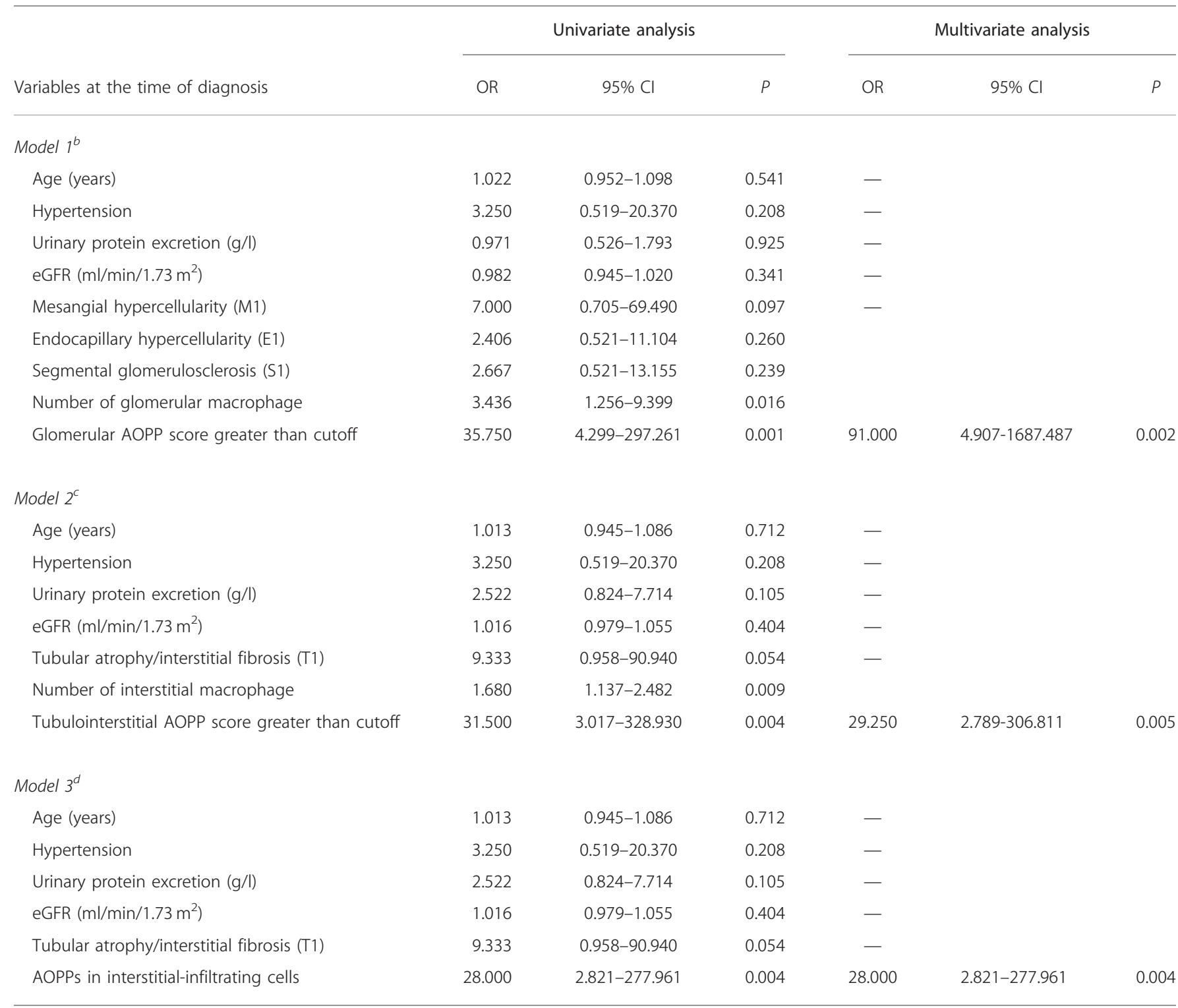

Abbreviations: OR, odds ratio; $\mathrm{Cl}$, confidence interval.

${ }^{a} N=30$, progression of renal fibrosis was defined as an increase in glomerular sclerosis score or tubulointerstitial fibrosis index $\geq 0.5$ at the repeat biopsy.

${ }^{b}$ Prediction of glomerular AOPPs for progression of glomerular sclerosis.

CPrediction of tubulointerstitial AOPPs for progression of tubulointerstitial fibrosis.

${ }^{\mathrm{d}}$ Prediction of AOPPs in interstitial-infiltrating cells for progression of tubulointerstitial fibrosis.

progressive course, but such a heavy proteinuria is infrequently present at clinical onset of IgAN or at initial biopsy. ${ }^{37,38}$ Proteinuria was $\geq 2 \mathrm{~g}$ /day at diagnosis of IgAN in only $17 \%$ of our patients. Likewise, impairment of renal function, once established, usually relentlessly progresses towards ESRD. Therefore, these risk factors, as reported in most studies, are somewhat insensitive. It would be much more relevant to detect patients at risk of developing progressive disease at an earlier stage of IgAN, when eGFR is still normal or nearly normal and when therapeutic intervention has the best chances of stopping or delaying the progression. We found that renal AOPP expression, at the time of diagnosis, was predictive for progression of renal fibrosis in IgAN patients with low-grade lesion, providing new information for early risk stratification in patients with IgAN.

Our study has a limitation. This is a single-center study with relatively small sample size and not including patients with nephrotic range proteinuria. The number of patients who received repeat renal biopsy is limited. Therefore the 
finding of the study needs to be further validated in multicenter and large sample cohort study.

In conclusion, our results validate the early predictive value of renal AOPP accumulation on renal progression in IgAN patients with low-grade lesion and normal renal function. This emphasizes the need for including oxidative stress biomarkers to early identify disease progression, prognosis, and probably therapeutic response in IgAN.

Supplementary Information accompanies the paper on the Laboratory Investigation website (http://www.laboratoryinvestigation.org)

\section{ACKNOWLEDGMENTS}

This study was supported by the National 973 program (No. 2012CB517700 and 2011CB504005), National Nature and Science Grants (No.U0932002), the Public Welfare and Health Sector Research Project (201002010), and National Key Technologies R\&D Program (2011BAI10B02) to Dr Fan Fan Hou and National Nature and Science Grants (No. 81270826) to Dr Jun Wang.

\section{DISCLOSURE/CONFLICT OF INTEREST}

The authors declare no conflict of interest.

1. Coppo R, D'Amico G. Factors predicting progression of $\lg A$ nephropathies. J Nephrol 2005;18:503-512.

2. Falk RJ, Glassock RJ. IgA nephropathy and Henoch-Schonlein purpura. Nephrology Self-Assessment Program 2006;5:361.

3. Komatsu $\mathrm{H}$, Fujimoto $\mathrm{S}$, Hara $\mathrm{S}$, et al. Relationship between serum $\lg A / C 3$ ratio and progression of IgA nephropathy. Intern Med 2004; 43:1023-1028.

4. Lai $\mathrm{FM}$, Szeto CC, Choi PCL, et al. Characterization of early $\lg \mathrm{A}$ nephropathy. Am J Kidney Dis. 2000;36:703-708.

5. Coppo R, Camilla R, Amore A, et al. Oxidative stress in $\lg A$ nephropathy. Nephron Clin Pract 2010;116:c196-c198.

6. Li HY, Hou FF, Zhang $X$, et al. Advanced oxidation protein products accelerate renal fibrosis in a remnant kidney model. J Am Soc Nephrol 2007; 18:528-538.

7. Floege J, Eitner F. Current therapy for IgA nephropathy. J Am Soc Nephrol 2011;22:1785-1794.

8. Kidney Disease: Improving Global Outcomes (KDIGO) Glomerulonephritis Work Group. KDIGO clinical practice guideline for glomerulonephritis. Kidney Int Suppl 2012;2:139-274.

9. Kidney Disease: Improving Global Outcomes (KDIGO) CKD Work Group. KDIGO 2012 clinical practice guideline for the evaluation and management of chronic kidney disease. Kidney Int Suppl 2013;3:1-150.

10. Radford MJ, Donadio JJ, Bergstralh EJ, et al. Predicting renal outcome in IgA nephropathy. J Am Soc Nephrol 1997;8:199-207.

11. Roberts IS, Cook HT, Troyanov S, et al. The Oxford classification of IgA nephropathy: pathology definitions, correlations, and reproducibility. Kidney Int 2009;76:546-556.

12. Liu $B$, Hou $X$, Zhou $Q$, et al. Detection of advanced oxidation protein products in patients with chronic kidney disease by a novel monoclonal antibody. Free Radic Res 2011;45:662-671.

13. Xavier LL, Viola GG, Ferraz AC, et al. A simple and fast densitometric method for the analysis of tyrosine hydroxylase immunoreactivity in the substantia nigra pars compacta and in the ventral tegmental area. Brain Res Protoc 2005;16:58-64.

14. Cao $\mathrm{W}, \mathrm{Xu} J$, Zhou ZM, et al. Advanced oxidation protein products activate intrarenal renin-angiotensin system via a CD36-mediated redox-dependent pathway. Antioxid Redox Signal 2013;18:19-35.
15. Liu SX, Hou FF, Guo ZJ, et al. Advanced oxidation protein products accelerate atherosclerosis through promoting oxidative stress and inflammation. Arterioscler Thromb Vasc Biol 2006;26:1156-1162.

16. Levey AS, Stevens $L A$, Schmid $C H$, et al. A new equation to estimate glomerular filtration rate. Ann Intern Med 2009;150:604-612.

17. Yamamoto T, Noble NA, Miller DE, et al. Sustained expression of TGFbeta 1 underlies development of progressive kidney fibrosis. Kidney Int 1994;45:916-927.

18. Ruster C, Wolf G. Renin-angiotensin-aldosterone system and progression of renal disease. J Am Soc Nephrol 2006;17:2985-2991.

19. Barbour SJ, Reich HN. Risk stratification of patients with IgA nephropathy. Am J Kidney Dis 2012;59:865-873.

20. Berthoux F, Mohey $\mathrm{H}$, Laurent $\mathrm{B}$, et al. Predicting the risk for dialysis or death in IgA nephropathy. J Am Soc Nephrol 2011;22:752-761.

21. Szeto CC, Lai FM, To KF, et al. The natural history of immunoglobulin a nephropathy among patients with hematuria and minimal proteinuria. Am J Med 2001;110:434-437.

22. Lai FM, Szeto CC, Choi PC, et al. Primary IgA nephropathy with low histologic grade and disease progression: is there a 'point of no return'? Am J Kidney Dis 2002;9:401-406.

23. Lee $S B$, Kalluri R. Mechanistic connection between inflammation and fibrosis. Kidney Int Suppl 2010;119:S22-S26.

24. Farris $A B$, Colvin RB. Renal interstitial fibrosis: mechanisms and evaluation. Curr Opin Nephrol Hypertens 2012;21:289-300.

25. Descamps-Latscha B, Witko-Sarsat V, Nguyen-Khoa T, et al. Early prediction of IgA nephropathy progression: proteinuria and AOPP are strong prognostic markers. Kidney Int 2004;66:1606-1612.

26. Valli $A$, Suliman ME, Meert $N$, et al. Overestimation of advanced oxidation protein products in uremic plasma due to presence of triglycerides and other endogenous factors. Clin Chim Acta 2007; 379:87-94.

27. Witko-Sarsat V, Friedlander M, Capeillere-Blandin C, et al. Advanced oxidation protein products as a novel marker of oxidative stress in uremia. Kidney Int 1996;49:1304-1313.

28. Witko-Sarsat V, Friedlander M, Nguyen KT, et al. Advanced oxidation protein products as novel mediators of inflammation and monocyte activation in chronic renal failure. J Immunol 1998;161: 2524-2532.

29. Guo ZJ, Niu HX, Hou FF, et al. Advanced oxidation protein products activate vascular endothelial cells via a RAGE-mediated signaling pathway. Antioxid Redox Signal 2008;10:1699-1712.

30. Zhou LL, Cao W, Xie C, et al. The receptor of advanced glycation end products plays a central role in advanced oxidation protein productsinduced podocyte apoptosis. Kidney Int 2012;82:759-770.

31. Wei XF, Zhou QG, Hou FF, et al. Advanced oxidation protein products induce mesangial cell perturbation through PKC-dependent activation of NADPH oxidase. Am J Physiol Renal Physiol 2009:296:F427-F437.

32. Haugen $E$, Nath KA. The involvement of oxidative stress in the progression of renal injury. Blood Purif 1999;17:58-65.

33. Shah SV, Baliga R, Rajapurkar M, et al. Oxidants in chronic kidney disease. J Am Soc Nephrol 2007;18:16-28.

34. Wakai K, Kawamura T, Endoh M, et al. A scoring system to predict renal outcome in IgA nephropathy: from a nationwide prospective study. Nephrol Dial Transplant 2006;21:2800-2808.

35. Xie J, Kiryluk K, Wang W, et al. Predicting progression of $\lg A$ nephropathy: new clinical progression risk score. PLoS One 2012;7: e38904.

36. Reich HN, Troyanov S, Scholey JW, et al. Remission of proteinuria improves prognosis in IgA nephropathy. J Am Soc Nephrol 2007;18:3177-3183.

37. D'Amico G. Natural history of idiopathic IgA nephropathy: role of clinical and histological prognostic factors. Am J Kidney Dis 2000;36: 227-237.

38. Ruggenenti P, Perna A, Gherardi G, et al. Chronic proteinuric nephropathies: outcomes and response to treatment in a prospective cohort of 352 patients with different patterns of renal injury. Am J Kidney Dis 2000;35:1155-1165. 